\title{
Transient disruption of M1 during response planning impairs subsequent offline consolidation
}

\author{
Nichola Rice Cohen · Emily S. Cross • \\ Nicholas F. Wymbs $\cdot$ Scott T. Grafton
}

Received: 18 August 2008 / Accepted: 30 April 2009 / Published online: 22 May 2009

(C) The Author(s) 2009. This article is published with open access at Springerlink.com

\begin{abstract}
Transcranial magnetic stimulation (TMS) was used to probe the involvement of the left primary motor cortex (M1) in the consolidation of a sequencing skill. In particular we asked: (1) if M1 is involved in consolidation of planning processes prior to response execution (2) whether movement preparation and movement execution can undergo consolidation independently and (3) whether sequence consolidation can occur in a stimulus specific manner. TMS was applied to left M1 while subjects prepared left hand sequential finger responses for three different movement sequences, presented in an interleaved fashion. Subjects also trained on three control sequences, where no TMS was applied. Disruption of subsequent consolidation was observed, but only for sequences where subjects had been exposed to TMS during training. Further, reduced consolidation was only observed for movement preparation, not movement execution. We conclude that left M1 is causally involved in the consolidation of effective response planning for left hand movements prior to
\end{abstract}

N. R. Cohen · E. S. Cross · N. F. Wymbs · S. T. Grafton HB 6162 Moore Hall,

Department of Psychological and Brain Sciences,

Center for Cognitive Neuroscience,

Dartmouth College, Hanover, NH 03755, USA

e-mail: njrice@brandeis.edu

N. R. Cohen

Volen Center for Complex Systems, Brandeis University,

MS013, 415 South Street, Waltham, MA 02454-9110, USA

N. F. Wymbs · S. T. Grafton ( $ه)$

Department of Psychology, University of California,

Santa Barbara, Santa Barbara, CA 93106-9660, USA

e-mail: grafton@psych.ucsb.edu response execution, and mediates consolidation in a sequence specific manner. These results provide important new insights into the role of M1 in sequential memory consolidation and sequence response planning.

Keywords Contextual interference $\cdot$ Primary motor cortex $\cdot$ Sequence learning $\cdot$ Offline learning $\cdot$ Procedural memory

\section{Introduction}

The role of the primary motor cortex (M1) in motor execution has been long established. However, it is now known that this region is more than a simple "executor" of action. For example, several functional neuroimaging studies in humans have established activity in M1 during motor preparation/planning (Richter et al. 1997; Zang et al. 2003). It has also been demonstrated that M1 is plastic, undergoing reorganization during motor skill learning (for review, see Sanes and Donoghue 2000; Ungerleider et al. 2002). Recently, a great deal of research has focused on the role of M1 in the consolidation of a skill (Muellbacher et al. 2002; Baraduc et al. 2004; Robertson et al. 2005; Richardson et al. 2006; Hadipour-Niktarash et al. 2007). Consolidation is a behavioural phenomenon that occurs between practice sessions. It can involve both offline learning (between session improvements), or memory stabilization (reduction in fragility of a memory) (Robertson et al. 2004). In this study, we attempted to further characterize the role of M1 in consolidation of a motor skill.

Transcranial magnetic stimulation (TMS) studies have highlighted the causal involvement of the primary motor cortex (M1) in procedural memory consolidation (Muellbacher et al. 2002; Robertson et al. 2005). These studies have shown 
that TMS to M1 disrupts consolidation if it is applied within 6 (Muellbacher et al. 2002) or $2 \mathrm{~h}$ (Robertson et al. 2005) of initial skill acquisition. It has also been shown that TMS to left M1 before learning can block subsequent consolidation (Richardson et al. 2006). These studies used repetitive TMS (rTMS), the effects of which last for a period of time after stimulation (e.g. see Muellbacher et al. (2000)), making it impossible to determine when M1 becomes involved in consolidation and how it is processing stimuli. A recent study used single pulse TMS to explore the chronometry of M1 in skill learning and retention (Hadipour-Niktarash et al. 2007). This study showed that M1 is involved in retention of arm movement adaptation when applied immediately (but not $700 \mathrm{~ms}$ ) following a movement, illustrating that M1 contributes to consolidation in a time-dependent manner. While these studies have shown spatial and temporal specificity of M1 in consolidation, several questions remain unanswered, in particular: (1) Is M1 involved in consolidation of planning processes that occur prior to response execution? (2) Can different aspects of a skill undergo consolidation independently? (3) Does M1 contribute to consolidation in a stimulus specific manner?

A recent functional imaging study from our group (Cross et al. 2007) exploited a paradigm known as contextual interference (CI) to investigate the neural substrates of skill acquisition and retention. The CI effect refers to a situation whereby performance of a new skill is typically better during initial skill acquisition when one uses a blocked practice schedule for different trial types compared to a practice schedule where all trials are randomly distributed. The key feature of the CI effect is that consolidation, measured with a retention test from 4 to $24 \mathrm{~h}$ after initial training, is better for the trials trained on a random practice schedule (Battig 1972; Shea and Morgan 1979). Cross et al. (2007) showed that imaging data reflecting motor preparation (i.e. when an instruction is transformed to an intended motor act) revealed stronger activity in a network of sensorimotor and premotor regions, including left (ipsi-lateral) M1, for those trials learned under random training conditions. A retention test confirmed greater consolidation for these random sequences, supporting a role of M1 in sequential memory consolidation and the $\mathrm{CI}$ effect. Interestingly, the results showed that the role of left M1 in consolidation for the random group is related to the pre-movement interval, and not related to the movement per se. This finding is supported other functional imaging data demonstrating a role of left M1 during movement preparation (Richter et al. 1997; Zang et al. 2003).

Here we attempted to further characterize the role of left M1 during movement preparation in subsequent sequence consolidation. In particular, we asked if left M1 is causally involved in sequential memory consolidation during movement preparation, and whether this involvement is sequence specific. In addition, we asked whether the left M1 activity observed during movement preparation for sequences learned under random training conditions (Cross et al. 2007) is responsible for consolidation of movement preparation and movement execution differentially.

\section{Method}

Subjects

Ten participants took part in this study after providing written informed consent (eight females, two males; mean age \pm standard deviation (SD), $23 \pm 3.94$ years old). Dartmouth Institutional Review Board approval was granted for all procedures. All subjects were right handed, as determined using the Edinburgh Handedness Inventory (Oldfield 1971).

\section{Materials}

A total of six different stimuli were used in the experiment, comprising four-number sequences consisting of the numbers 1 through 4 . Stimuli did not contain runs of three or more consecutive numbers (e.g. 4123). Sequences 1234 and 4321 were used as practice stimuli. Of the six different stimuli selected, three were assigned to the TMS block, three were assigned to the no TMS block; the sequences assigned to each block were counterbalanced across subjects. Custom written MATLAB scripts were used to present stimuli to participants. Each stimulus was presented to subjects on a computer monitor for a total of $200 \mathrm{~ms}$, after which time the screen went blank for $2 \mathrm{~s}$, allowing time for the subjects to make their response. After the $2 \mathrm{~s}$ response window subjects received feedback as to their accuracy in reproducing the stimuli (correct/incorrect). Feedback information was available on the screen for $1 \mathrm{~s}$, after which time the next trial commenced. The presentation of stimuli is depicted in Fig. 1.

\section{Procedure}

The experiment consisted of two sessions: (1) training session; (2) retest session. Each session was separated by $24 \mathrm{~h}$ and was carried out in the morning, between 8 am and noon. The timing of the task was important as previous studies have demonstrated that different aspects of a skill undergo consolidation over day and over night (Cohen et al. 2005; Cohen and Robertson 2007) and we wanted to ensure that all subjects were consolidating the skill in the same fashion. Subjects were seated $57 \mathrm{~cm}$ in front of a computer monitor and asked to place their left hand on the keyboard, with their fingers positioned over the keys A, S, 


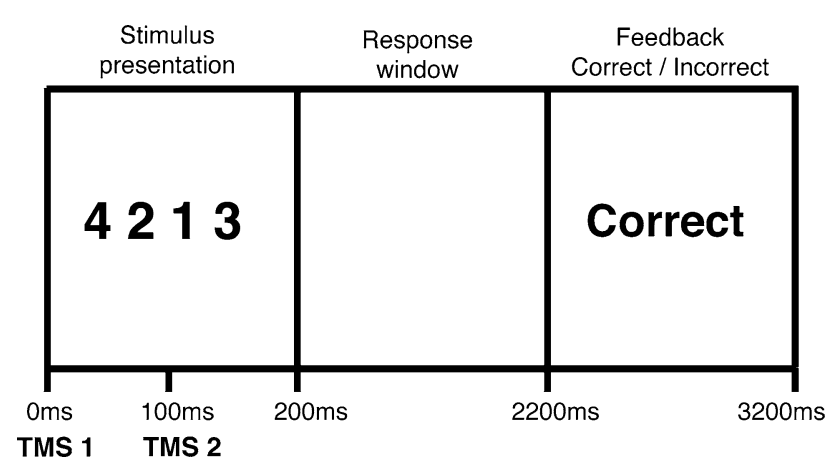

Fig. 1 Methods. Figure depicts the presentation of one trial. The sequence is presented on the screen for $200 \mathrm{~ms}$. When TMS is delivered the first pulse is delivered simultaneous with presentation of the sequence (TMS 1), with the second pulse occurring $100 \mathrm{~ms}$ later (TMS 2 ). The screen goes blank for $2 \mathrm{~s}$ allowing subjects to respond. This is followed by accuracy feedback, which is presented for $1 \mathrm{~s}$

D, F (such that their little finger was over A, ring finger over S, middle finger over $\mathrm{D}$ and index finger over F). Asking subjects to respond with their non-dominant left hand while we stimulated left M1 allowed us to specifically probe the involvement of M1 in response planning, without impairing response execution due to TMS-induced contraction of the intrinsic hand muscles of the contra-lateral hand. Subjects were instructed that a sequence of four numbers (comprising the numbers 1, 2, 3,4) would appear briefly on the screen, and that their task was to reproduce the sequence of numbers using the keys $\mathrm{A}, \mathrm{S}, \mathrm{D}, \mathrm{F}$ (which corresponded to the numbers $1,2,3,4)$. The cue to respond appeared as soon as the numbers disappeared off the screen. Participants were instructed to make their response as quickly and accurately as possible. Before beginning each session, subjects were given a block of practice trials, where they practiced the sequences $1,2,3,4$ and 4, 3, 2, 1 .

\section{Training session}

During the training session, subjects completed two blocks of trials: (1) TMS block; (2) no-TMS block. The presentation of blocks was counterbalanced across subjects. For the TMS block, TMS was delivered to the primary motor cortex on every trial (see below for details) simultaneous with presentation of the sequence. For the no TMS block, no TMS was delivered. Each block comprised 54 trials, made up of 18 repetitions of three different sequences. Within each block the trials were presented in a random order. Subjects were allowed a short break after each set of 18 trials.

\section{Retest session}

In the retest session subjects viewed: (1) TMS sequences; (2) No-TMS sequences. These sets of sequences were presented to subjects in separate blocks, the order of which was counterbalanced across subjects. It is important to note that no TMS was delivered in the retest session (the TMS sequences refer to the sequences where they had received TMS during the training session). For the no-TMS block these sequences were the same three sequences from the training session where subjects had received no TMS. For the TMS block the sequences were the three sequences where subjects had received TMS during the training session. Each block comprised a total of 18 trials, made up of six repetitions of three sequences. Within each block of 18 trials, half the trials were presented in a random fashion, and half were presented in a massed fashion, the order of which was counterbalanced across subjects.

\section{Localization of brain site and TMS}

One cortical site was chosen for stimulation: primary motor cortex of the left hemisphere. This site was localised functionally for all subjects, with the coil being placed tangentially over the skull with the handle pointing backwards. The coil was moved over the surface of the skull until a visible contraction of the intrinsic hand muscles in the right hand was observed. The intensity of the stimulation was gradually reduced to each subject's motor threshold. Motor threshold was determined as the intensity required to produce a visible contraction of the intrinsic hand muscles on five out of ten consecutive trials.

A Neotonus PNS stimulator (model no. N-0233-A$110 \mathrm{~V}$ ) with an air cooled iron-core butterfly shaped coil was used to administer TMS. Pulse duration for this stimulator and head coil is $180 \mu$ s (at $100 \%$ of operating power). TMS was applied at $110 \%$ of motor threshold, which corresponded to an average stimulator output of $54.9 \%$ (range $=48-59 \%)$. Ear plugs were provided to dampen the noise associated with the discharge from the TMS coil. For the TMS block, subjects received two pulses of TMS to primary motor cortex on every trial, the first pulse was applied simultaneous with the presentation of the stimulus on the screen, and the second pulse occurred $100 \mathrm{~ms}$ after the first. Such a method of applying TMS has been described elsewhere (Rice et al. 2006, 2007; Cohen et al. 2009), and was utilized in to disrupt processing within M1 during the entire stimulus viewing period (for discussion, see Rice et al. 2006). It should be noted that two pulses of TMS on every trial, with a $100 \mathrm{~ms}$ inter-stimulation interval and a $3 \mathrm{~s}$ interval between pairs of trials, would not be expected to cause sustained alteration in cortical activity that is usually associated with rTMS. A review of all our studies using these stimulation parameters has shown that the effects of our double-pulsed TMS last approximately $350 \mathrm{~ms}$ after administration of the pulses (Cohen et al. 2009). 
Statistical analysis

Trials were coded as incorrect if the wrong sequence was reproduced, if subjects commenced their reproduction while the stimulus was on the screen, or if subjects took more than $2 \mathrm{~s}$ to respond. Only correct trials were included in the analysis. For the training session this involved $91.12 \%$ of trials, and for the retest session this involved $91.67 \%$ of trials. Our dependent variables included response planning time and response execution time. Response planning time refers to the time subjects make the first key press, minus the disappearance of the stimuli from the screen. This pre-response interval may involve multiple steps, including selection, planning and preparation (Hoshi and Tanji 2007). Response execution time refers to the time subjects make their final key press, minus the time they make their first key press. For analysis of the training data, which provides a measure of skill acquisition, trials were binned into three groups of 18 trials (to make analysis of the training and retest data comparable). A repeated measures ANOVA was performed with the within-subjects factors of sequence (TMS versus no TMS sequences) and bin (three groups of 18 consecutive trials). To compute skill consolidation, we compared test to retest data. Here we computed the mean of the last 18 trials in the training data for each condition, and then subtracted this from each of the corresponding trials in the retest session. All data is provided in Table 1.

\section{Results}

Skill acquisition

To measure skill acquisition we analysed performance during the initial training session. Analysis of response planning times in the initial training data showed a significant effect of bin (with each bin representing 18 consecutive trials) $\left(F_{(2,18)}=9.095, P=0.002\right)$. Importantly, no significant effect of TMS was observed within the training session $\left(F_{(1,9)}=0.862, P=0.377\right)$, and no significant interaction was present $\left(F_{(2,18)}=0.084, \quad P=0.920\right)$. Analysis of response execution times during the initial training session also revealed a significant effect of bin $\left(F_{(2,18)}=7.032\right.$, $P=0.006)$, and no significant effect of TMS $\left(F_{(1,9)}=1.600\right.$, $P=0.238)$, or interaction $\left(F_{(2,18)}=1.230, P=0.316\right)$. Overall the analysis of the training data shows that subjects learned the sequences, but TMS had no effect on skill acquisition. Data from the training session in depicted in Fig. 2a.

\section{Consolidation}

To assess consolidation we compared performance at retest to performance at the end of training. We determined the mean of the last 18 trials in the training data for each condition (TMS and no TMS), and then subtracted this value from each of the corresponding trials in the retest session; a positive value reflects offline learning. This analysis is depicted in Fig. 2b. Analysis of response planning time data revealed a significant effect of sequence type $\left(F_{(1,9)}=5.757, P=0.04\right)$, with significantly greater consolidation for no TMS sequences compared to TMS sequences. Analysis of response execution time data revealed no significant effects of sequence type $\left(F_{(1,9)}=0.024, P=0.881\right)$, with subjects showing no significant differences in the consolidation of the TMS and the no TMS sequences.

\section{Discussion}

Our results show that when subjects were not exposed to TMS, they showed offline learning as measured by a faster response planning time of the newly acquired sequencing skill. Importantly, when training was accompanied by the application of TMS to left M1, with stimulation occurring immediately prior to the execution of sequences, there was a disruption of the offline gains measured as faster response planning. That is, TMS disrupted consolidation for learning related to sequence preparation. No such TMS effects were observed for sequence execution. Our results indicate that: (1) there is a role of left M1 in sequence consolidation that

Table 1 Summarizes means and standard deviations for each condition

\begin{tabular}{|c|c|c|c|c|c|c|c|c|c|c|c|}
\hline & & \multicolumn{6}{|c|}{ Training session } & \multicolumn{2}{|c|}{ Retest session } & \multicolumn{2}{|c|}{ Consolidation } \\
\hline & & \multicolumn{3}{|c|}{ TMS trials } & \multicolumn{3}{|c|}{ No TMS trials } & \multirow{2}{*}{$\begin{array}{l}\text { TMS } \\
\text { trials }\end{array}$} & \multirow{2}{*}{$\begin{array}{l}\text { No TMS } \\
\text { trials }\end{array}$} & \multirow{2}{*}{$\begin{array}{l}\text { TMS } \\
\text { trials }\end{array}$} & \multirow{2}{*}{$\begin{array}{l}\text { No TMS } \\
\text { trials }\end{array}$} \\
\hline & & Bin 1 & Bin 2 & Bin 3 & Bin 1 & Bin 2 & Bin 3 & & & & \\
\hline \multirow[t]{2}{*}{ Planning time (ms) } & Mean & 772.74 & 692.72 & 679.92 & 803.31 & 741.84 & 724.91 & 697.28 & 648.59 & -17.36 & 76.31 \\
\hline & Standard deviation & -150.91 & 110.04 & 95.65 & 141.66 & 143.02 & 171.41 & 134.59 & 133.45 & 97.85 & 107.64 \\
\hline \multirow[t]{2}{*}{ Execution time (ms) } & Mean & 955.59 & 865.44 & 836.48 & 916.19 & 821.74 & 836.76 & 822.13 & 826.24 & 14.35 & 10.52 \\
\hline & Standard deviation & 240.61 & 233.01 & 258.78 & 279.85 & 281.91 & 311.16 & 249.97 & 267.58 & 79.77 & 117.20 \\
\hline
\end{tabular}


Fig. 2 Graph depicts mean response planning time and standard error (top panel) and mean movement execution time and standard error (bottom panel) for: a the initial training session (data is binned into 3 groups of 18 consecutive trials), reflecting skill acquisition; $\mathbf{b}$ the difference in performance on last trials of the test session, minus performance on the retest session, reflecting skill consolidation. $* P<0.05$
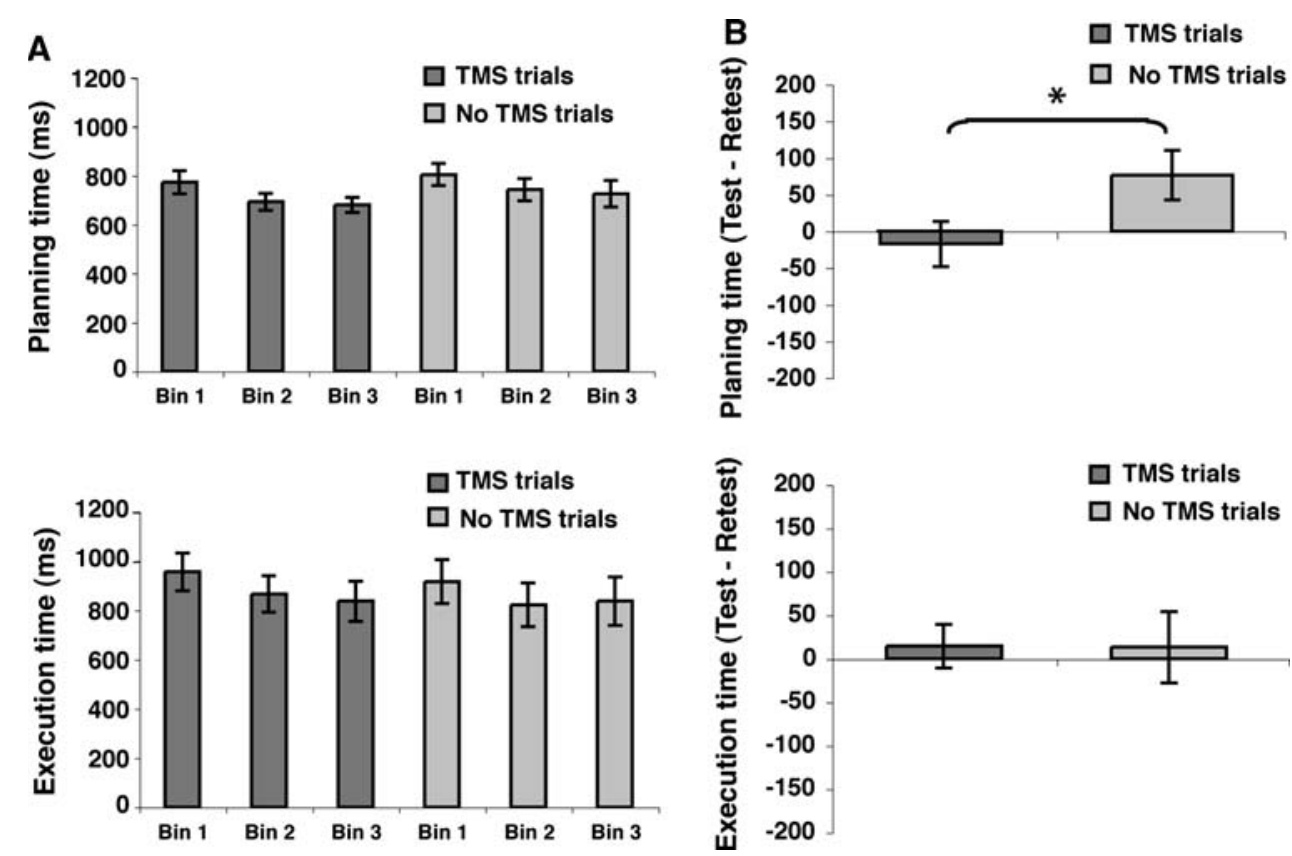

originates during the pre-response interval; (2) left M1 is involved in the consolidation of sequences in a sequence specific manner; (3) left M1 is involved in planning sequential responses for the left hand and this aspect of behavior can undergo consolidation independently from response execution.

While several studies have provided important insights into the critical time windows when M1 is involved in sequence consolidation, these studies have focused mainly on processes occurring after training has been completed. It is less clear if the conditions of training and events during the training period will influence consolidation processes in M1. In particular, TMS applied to M1 immediately following initial skill acquisition disrupts memory consolidation (Muellbacher et al. 2002; Baraduc et al. 2004; Robertson et al. 2005), but only if applied within $6 \mathrm{~h}$ (Muellbacher et al. 2002), and as little as $2 \mathrm{~h}$ (Robertson et al. 2005) of initial skill acquisition, revealing that the involvement of M1 in consolidation for sequence execution is temporally specific. While a recent study has demonstrated that TMS to left M1 before learning can block subsequent consolidation of a motor adaptation task, whilst having no influence on initial skill acquisition (Richardson et al. 2006) it was unclear if such findings could be extended to a sequential learning task. Also, the use of rTMS made it impossible to characterize the interaction of the timing of the TMS pulse to $\mathrm{M} 1$ and effects on planning versus execution processes. While findings from a single pulse TMS study have shown that M1 is involved in consolidation when applied immediately after movement execution (Hadipour-Niktarash et al. 2007), our results show for the first time that there is also a sensitivity of M1 consolidation to TMS during training when it is applied prior to movement execution, during sequence response planning.

One other important finding was that consolidation occurred in a sequence specific manner. In other words, we disrupted the process of consolidation only for sequences where subjects had been exposed to TMS during encoding, rather than interfering with consolidation for all learned sequences, highlighting the specificity of left M1 for learning and consolidating sequential sensory-motor responses. To our knowledge this is the first study to demonstrate that M1 contributes to consolidation in such a sequence specific manner, with previous studies disrupting consolidation for all components of the newly learned skill (Muellbacher et al. 2002; Baraduc et al. 2004; Robertson et al. 2005; Richardson et al. 2006; Hadipour-Niktarash et al. 2007).

Our results also provide some support for the idea that sequence response planning and execution involve dissociable networks, and further that these components of a skill can undergo consolidation independent of one another. Our results revealed that TMS only disrupted the consolidation of the sequence response planning component of the task but not movement execution. The idea that M1 is involved in sequence response planning has been suggested in previous neuroimaging studies (Richter et al. 1997; Zang et al. 2003; Cross et al. 2007). Due to the nature of functional imaging data, however, these studies have only provided correlative evidence of such involvement. By using TMS to transiently disrupt M1 during sequence response planning, we can attribute anatomy with function and establish that left M1 is necessary for consolidation of sequence response planning. 
Our results also add to the literature regarding the laterality of sequence learning. It has been shown that sequence learning with the non-dominant left hand causes a network of brain activations, largely lateralised to the left hemisphere (Grafton et al. 2002). In addition, mirror transformation of the learned sequence to the right hand results in activation in left motor cortex (Grafton et al. 2002). It has been shown that rTMS to left M1 disrupts offline improvements on a task performed with the right hand (Robertson et al. 2005). Our results extend this finding by showing that such a disruption can occur on a task performed with the ipsi-lateral (i.e. left) hand. It remains to be determined if right M1 also contributes to the process of consolidation, however our results, taken together with previous investigations, suggest that left M1 is necessary for consolidation, independent of hand used. A left hemisphere dominant network is commonly reported for the praxis system, however this likely engages more parietal and premotor circuits (Chao and Martin 2000; Haaland et al. 2000; Johnson-Frey et al. 2005). In addition, while imaging studies identify asymmetries of motor cortical activation for finger movements with the ipsi- or contra-lateral hand, with left M1 engaged more for ipsi-lateral (left-handed) movements (Kim et al. 1993), it remains unclear if activation of the left M1 for left hand movement is related to the actual planning or execution of a motor sequence. Our results suggest that the role of left M1 is specific to the consolidation of sequence response planning.

One might argue that the effects observed in our study can be generalized to other cortical areas or may be accounted for by distraction associated with a contraction of the intrinsic hand muscles in the contralateral hand (as TMS was applied at $110 \%$ of motor threshold). Our results cannot be accounted for by either of these explanations for several reasons. First, a role of M1 in sequential memory consolidation has been well established by others (Muellbacher et al. 2002; Robertson et al. 2005) using several control sites including occipital cortex, dorsolateral prefrontal cortex (Muellbacher et al. 2002) and a site $25 \mathrm{~mm}$ anterior to M1 (Robertson et al. 2005). These studies have established that the observed role of M1 in consolidation cannot be generalized to other cortical areas. We were interested in specifically characterizing this previously established role of M1 in consolidation, and therefore we had no reason to stimulate another cortical site. Second, we only applied TMS to M1 during the training session and our TMS induced effects were observed only on subsequent consolidation measures. If the application of TMS were having a generalized impact on performance, the effects would have been observed during the initial training session (which was the only time at which TMS was applied), and would not be restricted to consolidation. Also, we applied TMS to M1 only during the sequence response planning interval. Once again if our findings can be attributed to a generalized effect of TMS we would not expect the effects to be specific to response planning. Subjects also performed the task with their ipsilateral hand and all motor contractions were experienced by the contra-lateral hand only. The fact that we observed TMS induced effects only on retest (when no TMS was applied) and only on response planning measures, rules out a generalized TMS induced impairment in performance, and supports other studies showing that the involvement of M1 in consolidation cannot be generalized to other cortical areas (Muellbacher et al. 2002; Robertson et al. 2005).

In conclusion, our results show that applying double pulses of TMS to left M1 during the response planning interval, while subjects learn a set of different movement sequences, does not disrupt skill acquisition, but impairs subsequent memory consolidation of response planning of the sequences. These findings show that M1 contributes to consolidation of sequence response planning, and this is independent of the role of M1 in execution. Our findings also show that M1 contributes to consolidation in a sequence-specific manner; thus providing novel insights into when and how M1 contributes to procedural memory consolidation, which is important for models of plasticity.

Acknowledgments This work was supported by the National Science Foundation and PHS grants NS033504 and NS44393. The authors wish to thank Daniel Cohen for his thoughtful comments on an earlier version of this manuscript, and George Wolford for advice on statistical analysis.

Open Access This article is distributed under the terms of the Creative Commons Attribution Noncommercial License which permits any noncommercial use, distribution, and reproduction in any medium, provided the original author(s) and source are credited.

\section{References}

Baraduc P, Lang N, Rothwell JC, Wolpert DM (2004) Consolidation of dynamic motor learning is not disrupted by rTMS of primary motor cortex. Curr Biol 14:252-256

Battig WF (1972) Intratask interference as a source of facilitation in transfer and retention. In: Thompson RF, Voss JF (eds) Topics in learning and performance. Academic Press, New York

Chao LL, Martin A (2000) Representation of manipulable man-made objects in the dorsal stream. Neuroimage 12:478-484

Cohen DA, Robertson E (2007) Motor sequence consolidation: constrained by critical time windows or competing components. Exp Brain Res 177:440-446

Cohen DA, Pascual-Leone A, Press DZ, Robertson EM (2005) Offline learning of motor skill memory: a double dissociation of goal and movement. Proc Natl Acad Sci USA 102:1823718241

Cohen NR, Cross ES, Tunik E, Grafton ST, Culham JC (2009) Ventral and dorsal stream contributions to the online control of immediate and delayed grasping: a TMS approach. Neuropsychologia 47:1553-1562 
Cross ES, Schmitt PJ, Grafton ST (2007) Neural substrates of contextual interference during motor learning support a model of active preparation. J Cogn Neurosci 19:1854-1871

Grafton ST, Hazeltine E, Ivry RB (2002) Motor sequence learning with the nondominant left hand. A PET functional imaging study. Exp Brain Res 146:369-378

Haaland KY, Harrington DL, Knight RT (2000) Neural representations of skilled movement. Brain 123:2306-2313

Hadipour-Niktarash A, Lee CK, Desmond JE, Shadmehr R (2007) Impairment of retention but not acquisition of a visuomotor skill through time-dependent disruption of primary motor cortex. J Neurosci 27:13413-13419

Hoshi E, Tanji J (2007) Distinctions between dorsal and ventral premotor areas: anatomical connectivity and functional properties. Curr Opin Neurobiol 17:234-242

Johnson-Frey SH, Newman-Norlund R, Grafton ST (2005) A distributed left hemisphere network active during planning of everyday tool use skills. Cereb Cortex 15:681-695

Kim SG, Ashe J, Hendrich K, Ellermann JM, Merkle H, Ugurbil K, Georgopoulos AP (1993) Functional magnetic resonance imaging of motor cortex: hemispheric asymmetry and handedness. Science 261:615-617

Muellbacher W, Ziemann U, Boroojerdi B, Hallett M (2000) Effects of low-frequency transcranial magnetic stimulation on motor excitability and basic motor behavior. Clin Neurophysiol 111:10021007

Muellbacher W, Ziemann U, Wissel J, Dang N, Kofler M, Facchini S, Boroojerdi B, Poewe W, Hallett M (2002) Early consolidation in human primary motor cortex. Nature 415:640-644

Oldfield RC (1971) Assessment and analysis of handedness: Edinburgh inventory. Neuropsychologia 9:97-113
Rice NJ, Tunik E, Grafton ST (2006) The anterior intraparietal sulcus mediates grasp execution, independent of requirement to update: new insights from transcranial magnetic stimulation. J Neurosci 26:8176-8182

Rice NJ, Tunik E, Cross ES, Grafton ST (2007) Online grasp control is mediated by the contralateral hemisphere. Brain Res 1175:76-84

Richardson AG, Overduin SA, Valero-Cabre A, Padoa-Schioppa C, Pascual-Leone A, Bizzi E, Press DZ (2006) Disruption of primary motor cortex before learning impairs memory of movement dynamics. J Neurosci 26:12466-12470

Richter W, Andersen PM, Georgopoulos AP, Kim SG (1997) Sequential activity in human motor areas during a delayed cued finger movement task studied by time-resolved fMRI. Neuroreport $8: 1257-1261$

Robertson EM, Pascual-Leone A, Miall RC (2004) Current concepts in procedural consolidation. Nat Rev Neurosci 5:576-582

Robertson EM, Press DZ, Pascual-Leone A (2005) Off-line learning and the primary motor cortex. J Neurosci 25:6372-6378

Sanes JN, Donoghue JP (2000) Plasticity and primary motor cortex. Annu Rev Neurosci 23:393-415

Shea JB, Morgan RL (1979) Contextual interference effects on the acquisition, retention, and transfer of a motor skill. J Exp Psychol Learn Mem Cogn 5:179-187

Ungerleider LG, Doyon J, Karni A (2002) Imaging brain plasticity during motor skill learning. Neurobiol Learn Mem 78:553-564

Zang Y, Jia F, Weng X, Li E, Cui S, Wang Y, Hazeltine E, Ivry R (2003) Functional organization of the primary motor cortex characterized by event-related fMRI during movement preparation and execution. Neurosci Lett 337:69-72 